\title{
Prostate Adenocarcinoma without Neuroendocrine Differentiation
}

National Cancer Institute

\section{Source}

National Cancer Institute. Prostate Adenocarcinoma without Neuroendocrine

Differentiation. NCI Thesaurus. Code C150557.

A prostate adenocarcinoma characterized by the absence of focal or diffuse

neuroendocrine differentiation. 